\title{
Correction to: Outcomes of endoscopic treatment for malignant biliary obstruction in patients with surgically altered anatomy: analysis of risk factors for clinical failure
}

Takeshi Tomoda $^{1}$ (1) Hironari Kato ${ }^{1} \cdot$ Kazuya Miyamoto $^{1}$ - Akihiro Matsumi ${ }^{1} \cdot$ Eijiro Ueta $^{1} \cdot$ Yuuki Fujii ${ }^{1}$. Yousuke Saragai ${ }^{1}$ - Tatsuhiro Yamazaki ${ }^{1}$ - Daisuke Uchida ${ }^{1}$ - Kazuyuki Matsumoto ${ }^{1}$. Shigeru Horiguchi ${ }^{1}$. Koichiro Tsutsumi ${ }^{1} \cdot$ Hiroyuki Okada ${ }^{1}$

Published online: 6 February 2020

(c) Springer Science+Business Media, LLC, part of Springer Nature 2020

\section{Correction to: Surgical Endoscopy} https://doi.org/10.1007/s00464-020-07385-y

There are several places where the P-value and Odds ratio in Table 3 are incorrect: these are shown in the corrected Table 3.

The following two sentences related to Table 3 should be corrected:

1. In the Results section of the Abstract, the sentence

"In multivariate analysis, the presence of peritoneal dissemination (odds ratio, 7.3 ; 95\% confidence interval, $1.5-43.5, p=0.02$ ) was as an independent risk factor for clinical failure."

should read:

"In multivariate analysis, the presence of peritoneal dissemination (odds ratio, 6.8; 95\% confidence interval,
1.6-32.8, $p=0.008$ ) was as an independent risk factor for clinical failure."

2. In the last line of Clinical outcome section, the sentence

"In multivariate analysis, the presence of peritoneal dissemination (odds ratio, $7.3 ; 95 \%$ confidence interval, $1.5-43.5)(p=0.02)$ was as an independent risk factor for clinical failure."

should read:

"In multivariate analysis, the presence of peritoneal dissemination (odds ratio, 6.8; 95\% confidence interval, 1.6-32.8) ( $p=0.008)$ was as an independent risk factor for clinical failure."

The authors regret these errors.

The original article can be found online at https://doi.org/10.1007/ s00464-020-07385-y.

Takeshi Tomoda

tomotake79@yahoo.co.jp

\footnotetext{
Department of Gastroenterology and Hepatology,

Okayama University Hospital, 2-5-1 Shikata-cho, Kita-ku,

Okayama-city, Okayama 700-8558, Japan
} 
Table 3 Risk factor for clinical failure via endoscopic treatment of MBO in patients with surgically altered anatomy

\begin{tabular}{|c|c|c|c|c|c|c|c|c|}
\hline & \multirow{2}{*}{$\begin{array}{l}\text { Clinical failure } \\
n=14\end{array}$} & \multirow{2}{*}{$\begin{array}{l}\text { Clinical success } \\
n=31\end{array}$} & \multicolumn{3}{|c|}{ Univariate analysis } & \multicolumn{3}{|c|}{ Multivariate analysis } \\
\hline & & & OR & $95 \% \mathrm{CI}$ & $p$ value & OR & $95 \% \mathrm{CI}$ & $p$ value \\
\hline Age, $(>67$ years $), n(\%)$ & $7(50.0 \%)$ & $16(51.6 \%)$ & 0.9 & $0.3-3.3$ & 0.92 & & & \\
\hline Sex, male, $n(\%)$ & $8(57.1 \%)$ & $21(67.7 \%)$ & 0.6 & $0.2-2.3$ & 0.49 & & & \\
\hline \multicolumn{9}{|l|}{ Etiology of MBO, $n(\%)$} \\
\hline Malignant biliary disease & $4(28.6 \%)$ & $12(38.7 \%)$ & 0.6 & $0.1-2.4$ & 0.51 & & & \\
\hline Malignant pancreatic disease & $3(21.4 \%)$ & $10(32.3 \%)$ & 0.6 & $0.1-2.3$ & 0.45 & & & \\
\hline Gastric cancer & $6(42.9 \%)$ & $6(19.4 \%)$ & 3.1 & $0.8-12.9$ & 0.11 & & & \\
\hline Colon cancer & $0(0 \%)$ & $3(9.7 \%)$ & 0 & & 0.13 & & & \\
\hline Hepatocellular carcinoma & $1(7.1 \%)$ & $0(0 \%)$ & - & & 0.13 & & & \\
\hline Type of MBO, $n(\%)$ & & & 0.9 & $0.2-7.0$ & 0.90 & & & \\
\hline Primary disease & $2(14.3 \%)$ & $4(12.9 \%)$ & & & & & & \\
\hline Recurrent disease & $12(85.7 \%)$ & $27(87.1 \%)$ & & & & & & \\
\hline Liver metastasis & $2(14.3 \%)$ & $10(32.3 \%)$ & & & & & & \\
\hline Lymph node metastasis & $3(21.4 \%)$ & $4(12.9 \%)$ & & & & & & \\
\hline Local recurrence & $7(50.0 \%)$ & $13(41.9 \%)$ & & & & & & \\
\hline Location of MBO, $n(\%)$ & & & 2.2 & $0.6-8.3$ & 0.26 & & & \\
\hline Hilar or $\mathrm{HJ}$ anastomosis & $8(57.1 \%)$ & $23(74.2 \%)$ & & & & & & \\
\hline Distal & $6(42.9 \%)$ & $8(25.8 \%)$ & & & & & & \\
\hline Presence of peritoneal dissemination, $n(\%)$ & $8(57.1 \%)$ & $5(16.1 \%)$ & 6.9 & $1.7-31.3$ & 0.006 & 6.8 & $1.6-32.8$ & 0.008 \\
\hline \multicolumn{9}{|l|}{ Digestive reconstruction, $n(\%)$} \\
\hline Modified child's method & $3(21.4 \%)$ & $15(48.4 \%)$ & 0.3 & $0.1-1.1$ & 0.08 & 0.3 & $0.1-1.3$ & 0.12 \\
\hline RY gastorejejunostomy & $5(35.7 \%)$ & $8(25.8 \%)$ & 1.6 & $0.4-6.2$ & 0.50 & & & \\
\hline RY hepaticojejunostomy & $4(28.6 \%)$ & $5(16.1 \%)$ & 2.1 & $0.4-9.5$ & 0.34 & & & \\
\hline Billroth II with gastrectomy & $2(14.3 \%)$ & $3(9.7 \%)$ & 1.6 & $0.2-10.6$ & 0.66 & & & \\
\hline Presence of native papilla, $n(\%)$ & $7(50.0 \%)$ & $11(35.5 \%)$ & 1.8 & $0.5-6.7$ & 0.36 & & & \\
\hline Time from surgery to ERCP (> 505 days), $n(\%)$ & $5(35.7 \%)$ & $18(58.1 \%)$ & 0.4 & $0.1-1.4$ & 0.16 & & & \\
\hline T-Bil (> $2.5 \mathrm{mg} / \mathrm{dL}), n(\%)$ & $9(64.3 \%)$ & $13(41.9 \%)$ & 2.5 & $0.7-9.8$ & 0.16 & & & \\
\hline AST (> 80 IU/L), $n(\%)$ & $8(57.1 \%)$ & $14(45.2 \%)$ & 1.6 & $0.5-6.0$ & 0.46 & & & \\
\hline $\operatorname{ALT}(>72 \mathrm{IU} / \mathrm{L}), n(\%)$ & $8(57.1 \%)$ & $14(45.2 \%)$ & 1.6 & $0.5-6.0$ & 0.46 & & & \\
\hline$\gamma \mathrm{GTP}(>364 \mathrm{IU} / \mathrm{L}), n(\%)$ & $8(57.1 \%)$ & $13(41.9 \%)$ & 1.8 & $0.5-6.9$ & 0.34 & & & \\
\hline $\operatorname{ALP}(>1205 \mathrm{IU} / \mathrm{L}), n(\%)$ & $6(42.9 \%)$ & $15(48.4 \%)$ & 0.8 & $0.2-2.8$ & 0.73 & & & \\
\hline Type of scope, $n(\%)$ & & & 1.5 & $0.4-7.8$ & 0.59 & & & \\
\hline EI-580BT & $3(21.4 \%)$ & $9(29.0 \%)$ & & & & & & \\
\hline Other scope & $11(78.6 \%)$ & $22(71.0 \%)$ & & & & & & \\
\hline
\end{tabular}

$M B O$ malignant biliary obstruction, $H J$ hepaticojejunostomy, $R Y$ Roux-en-Y, $E R C P$ endoscopic retrograde cholangiopancreatography, $T B$ total bilirubin, $A S T$ aspartate aminotransaminase, $A L T$ alanine aminotransferase, $\gamma G T P$ g-glutamyl transpeptidase, $A L P$ alkaline phosphatase, $O R$ odds ratio, $C I$ confidence interval

Publisher's Note Springer Nature remains neutral with regard to jurisdictional claims in published maps and institutional affiliations. 